\title{
The Lennard-Jones Fluid in the Liquid-Vapour Critical Region
}

\author{
D.M. Heyes \\ Department of Physics, Royal Holloway \\ University of London, Egham, Surrey TW20 OEX, UK \\ E-mail: david.heyes@rhul.ac.uk
}

Received: 01 June 2015; accepted: 12 June 2015; published online: 23 August 2015

\begin{abstract}
The equation of state of the Lennard-Jones (LJ) fluid in the liquid-vapour (LV) critical region is investigated by Molecular Dynamics simulation (MD). The calculated pressure $(P)$ and chemical potential $(\mu)$ are, within the simulation statistics, flat at the critical temperature between LJ reduced densities of $c a$. 0.26 to 0.34 . The critical temperature, $T_{c}$, determined for an isotherm where $(\partial P / \partial \rho)_{T}=0$ and $(\partial \mu / \partial \rho)_{T}=0$, is shown to decrease with increasing system size and pure LJ potential interaction range, $r_{c}$, using a tapering function going to zero beyond $r_{c}$. The value of $T_{c}$ obtained by extrapolating the system size and $r_{c}$ to $\infty$ is $1.316 \pm 0.001$, which is statistically within the uncertainties of previous literature values. The percolation threshold separation, $r_{p}$, along the critical isotherm decreases monotonically with increasing density, $\rho$, and is for intermediate densities lower than that of the nearest equivalent hard-sphere system. The lines of constant percolation distance on the density-temperature projection of the phase diagram reveal a difference in qualitative behaviour, indicative of underlying structural differences on either side of the critical envelope. The mean square force in the critical region near to $T_{c}$ is linear in $\rho$. Probability distributions of the nearest neighbour distance, absolute particle force and potential energy per molecule are presented.
\end{abstract}

Key words: Lennard-Jones criticality, Molecular Dynamics simulation, percolation

\section{INTRODUCTION}

Many aspects of the fluid part of the phase diagram are still poorly understood, which includes the phase behaviour and equation of state in the liquid-vapour (LV) critical region. Andrews showed experimentally in 1869 that a coexisting liquid and vapour become a homogeneous supercritical fluid above a 'critical' temperature [1], and the 'supercritical fluid' state, (SCF) exists at all densities at temperatures above the critical temperature, [2]. In 1873 van der Waals proposed his now well-known cubic equation of state whose solution predicts that the liquid-vapour transition at the critical temperature, $T_{c}$, takes place at a point on the phase diagram specified by critical values, $\rho_{c}$, and, $P_{c}$, of density and pressure, respectively [3].

The existence of the liquid-vapour critical point is firmly established and taken as an accepted fact in the liquid state physical science and engineering literature. However, the liquid-vapour critical point has never been measured or achieved directly by experiment or molecular simulation but only inferred by extrapolation of coexistence data, even though very close to $T_{c}$ where there are no data points there is still a large density difference between the liquid and vapour densities (about $30 \%$ of $\rho_{c}$, [4]). It is already known that there is a weak pressure-density dependence in this region (e.g., $\left.\left|P-P_{c}\right| \propto\left|\rho-\rho_{c}\right|^{4},[5,6]\right)$ and it has been proposed a number of times over the last century that it is indeed flat (i.e., with zero slope), (e.g., see Refs. [7-10] and references quoted therein). This has been disputed (see for example, Refs. [11, 12]), and to date it appears that either of these two propositions could be correct. The 'flat top' proposal is that at $T_{c}$ there is a horizontal line joining the two coexisting phase properties, in which case $\Delta \rho>0$ and the surface tension, $\gamma>0$ at $T_{c}$. Indeed the experimental $P-\rho$ values along 
the critical isotherm are statistically flat in a density range on either side of the extrapolated critical density [13-15].

This work follows on from Ref. [10] in exploring the equation of state of the Lennard-Jones (LJ) fluid in the critical region using Molecular Dynamics (MD) simulation. The new features discussed here are a more systematic study of system size and interaction truncation distance effects. Further analysis of the structure of the fluid based on the Stillinger cluster construction procedure using a fixed neighbour distance for defining connectivity is made. The percolation properties of these clusters are analysed. The behaviour of other properties with density along the critical isotherm or neighbouring isotherms are also explored.

Molecular Dynamics (MD) has well-known limitations, including the small system size compared to typical macroscopic dimensions and short exploratory times compared with experiment. Despite these drawbacks, MD does have several advantages compared to experiment which are particularly useful in the present context. Because it can probe very short time scales, on the molecular scale, isotherms arbitrarily close above and even below $T_{c}$ can be mapped out by MD before any phase separation has time to occur, [16]. The isotherms in the critical region at a temperature below $T_{c}$ are of course metastable. In Ref. [10] a new procedure was proposed to determine the critical temperature $T_{c}$ (which I emphasise is not in dispute) as the temperature at which the slope of $P(\rho)$ is zero in the critical region of the phase diagram. The slope goes from being negative to positive on increasing temperature upwards through $T_{c}$. Another advantage of simulation compared to experiment is that the chemical potential can be computed directly, using for example, Widom's particle insertion method, [17] whereas this quantity has to be determined from experiment by integration of the measured density-pressure data (thereby introducing further statistical error). The flat top region at $T_{c}$ would be where at $T=T_{c}$ both $(\partial P / \partial \rho)_{T}=0$ and $(\partial \mu / \partial \rho)_{T}=0$ at all points within a certain density region in the neighbourhood of the assumed critical density.

The present study does not prove one way or the other if the critical point exists but it does provide further data and insights which may help ultimately to resolve this longstanding dispute. Inter alia some other aspects of the behaviour of the system in the region of criticality are brought out. It is emphasised that none of the figures which are discussed in the next section rely on the premise that a critical liquid-vapour critical point exists or not. They are totally independent and supplementary to this debate.

\section{SIMULATION DETAILS}

Molecular Dynamics (MD) simulations were carried out using the isotropic Lennard-Jones potential, $\phi(r)=$ $4 \epsilon\left[(\sigma / r)^{12}-(\sigma / r)^{6}\right]$, where $r$ is the distance between the centers of the two molecules. The quantities presented are given in the usual $\mathrm{LJ}$ reduced units, where $\sigma, \epsilon$ and the mass of the molecule are set to unity. The Lennard-Jones pair potential was used for the potential energy and force up to a distance of $r_{c}$ which ranges in various simulations between $2.5-4.5$. For larger distances than $r_{c}$ the interaction was gradually reduced to zero over a further 0.2 distance units using the Morris - Song tapering function [18]. This removes the discontinuity in the force and associated jump in potential energy which would occur if the LJ potential were simply truncated at $r_{c}$. This procedure ensures that the potential energy of the pairs for $r<r_{c}$ is the full Lennard-Jones potential, and not shifted downwards. There is no discontinuity in the potential or force experienced by the molecule as it crosses $r_{c}$ in this procedure. The usual long range correction formulas for the energy and pressure were applied, and based on the value of $r_{c}$ as the lower limit of the integral [19]. The critical temperature of the LJ system is known to be sensitive to truncation and various tapered modifications, [20,21], an issue which is studied further here.

The reduced number density is $\rho$, the reduced time step was $0.005 / \sqrt{ } T$ and post-equilibration simulations were typically conducted for $10^{6}$ time steps for each state point. The number of particles in the simulation cell ranged between 500 to 10,976 . The Lennard-Jones critical point parameters are, $T_{c}=1.3120(7),[22,23], \rho_{c}=0.316(1),[24]$ and $P_{c}=0.141(1),[25]$ respectively, where the number in brackets is the estimated uncertainty in the last digit. The triple point temperature and density are $c a .0 .69$ and 0.85 , respectively [26].

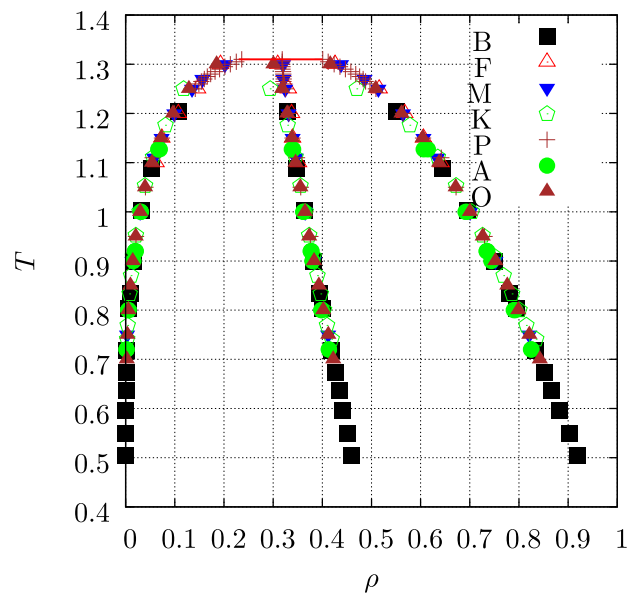

Fig. 1. Phase boundary between the LJ vapour and liquid phases obtained in various literature molecular simulation studies. The horizontal red line at the top of the coexistence envelope highlights where there are no data points. The central almost linear set of data points is the average of the vapour and liquid densities, with slope $-5.45 \pm 0.09$ and intercept $2.99 \pm 0.03$ which gives a critical density of $0.309 \pm 0.006$ taking a critical temperature of 1.312 . based on linear regression of all of the data. The key on the figure refers to the following references, [27] (B), [28] (F), [29] (K), [24] (M), [22] (P), [30] (A), and [31, 32] (O). 


\section{RESULTS AND DISCUSSION}

Fig. 1 traces out the LJ vapour and liquid coexisting densities as a function of temperature using data points taken from a number of molecular simulation studies, [22, 24, 27-32]. Note that there is a gap of almost 0.2 Lennard-Jones units between the co-existing vapour and liquid phases in the vicinity of $T_{c}$. The central almost linear set of data points is the average of the vapour and liquid densities, which can be seen to follow the law of rectilinear diameters, [33] quite well.

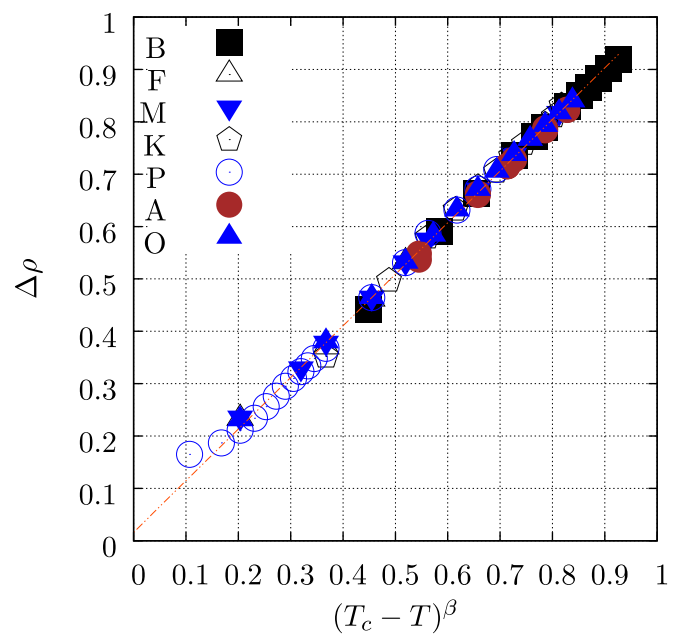

Fig. 2. The density difference between the coexisting Lennard-Jones liquid and vapour phases, $\Delta \rho=\rho_{l}-\rho_{v}$ as a function of $\left(T_{c}-T\right)^{\beta}$. Fitting to all of the data, the linear regression slope is $0.985 \pm 0.006$, the intercept is $0.016 \pm 0.008$, and $\beta=0.360 \pm 0.006$ (the mean field and non-classical values of $\beta$ are $1 / 2$ and $0.326 \pm 0.002$, respectively). The key on the figure identifying the reference sources is the same as for Fig. 1.

Fig. 2 presents the density difference between the coexisting liquid and vapour phases, $\Delta \rho=\rho_{l}-\rho_{v}$ as a function of $\left(T_{c}-T\right)^{\beta}$, using literature data. A least squares fit value for $\beta$ from these data is $0.360 \pm 0.006$, which is closer to the non-classical crossover value of $0.326 \pm 0.002$ than the van der Waals or mean field value of $\beta=1 / 2$. This is surprising as most of the data in the figure should comply with the van der Waals or mean field value of $\beta$, with the crossover to nonclassical behaviour taking place at temperatures very much closer to $T_{c}$. The wide temperature range where the exponent, $\beta$ is closer to the non-classical value rather than the mean field value might be considered contrary to expectations.

Fig. 3 presents the density difference between the coexisting liquid and vapour phases, $\Delta \rho=\left(\rho_{l}-\rho_{v}\right)^{\alpha}$ as a function of the surface tension, $\gamma_{S}$ using data taken from, $[20,22,30]$. A $\log -\log$ plot based on all the data has a slope of $\alpha \equiv \beta / \mu=$ $0.260 \pm 0.005$, which is close to the non-mean field value of 0.250 . This body of simulation data (at least) within statistics and on extrapolation, supports the existence of a critical point in predicting that there is no density difference between the liquid and vapour phases at $T_{c}$ and that the surface tension is zero in that limit too. As noted above, however, the data used for extrapolation terminates some distance from the $T_{c}$ limit, and its qualitative behaviour could change closer to $T_{c}$.

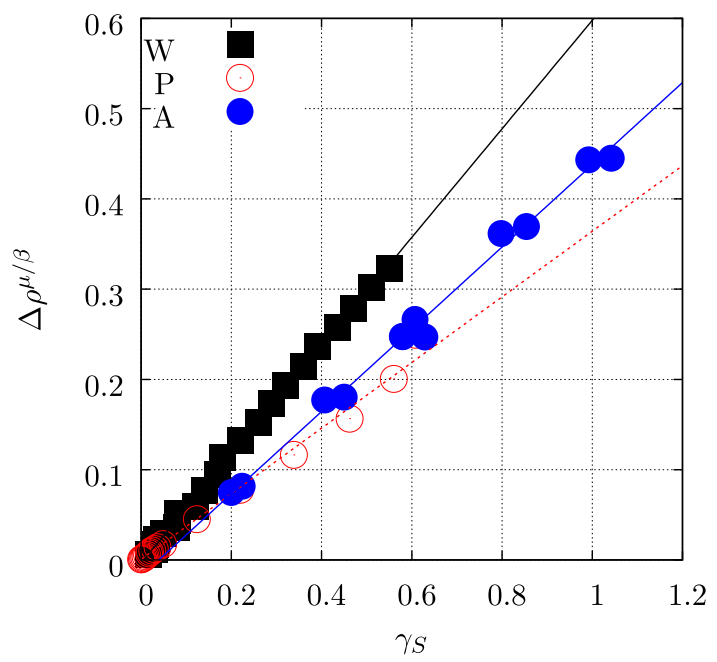

Fig. 3. The density difference between the coexisting Lennard-Jones liquid and vapour phases, $\Delta \rho=\rho_{l}-\rho_{v}$ as a function of the surface tension, $\gamma_{S}$. A log-log plot gives a slope of $\beta / \mu=0.260 \pm 0.005$, compared with the non-classical value of $0.326 / 1.303=0.250$. Key: References: [20] (W) (using a tapered LJ potential with a lower critical temperature than standard LJ), [22] (P), and [30] (A).

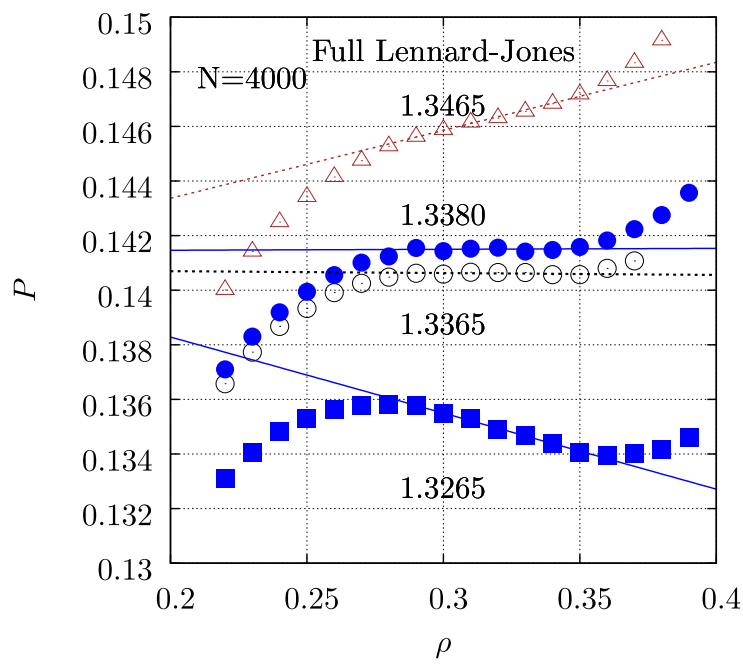

Fig. 4. Pressure, $P$ against $\rho$ for four isotherms, $T=$ $1.3265,1.3365,1.3380$ and 1.3465 (bottom to top curve, respectively) using $N=4000$ and $r_{c}=2.5$. The symbols are the Molecular Dynamics (MD) simulation data points and the lines are linear regression fits in the linear parts of $P-\rho$ for each temperature. The slopes are, $-0.028 \pm 0.001,0.0007 \pm 0.0001,0.0003 \pm 0.001$, and $0.025 \pm 0.001$, respectively, for the lines in ascending order. Therefore the critical temperature is $c a$. 1.337 which is where the slope is statistically zero in the density range, $\rho=0.28-0.35$. The pressure is equal to $0.1406(2)$ at this temperature and along the density line. 
Fig. 4 shows a plot of $P(\rho)$ in the critical density region from this work's simulations for four temperatures, using $N=4000$ molecules in the simulation cell, and $r_{c}=2.5$. The pressure is a linear function of density in the critical 'gap' for temperatures just above and below $T_{c}$. The critical temperature is defined as the temperature at which this slope is equal to zero in this density range. The value, $T_{c}=1.3365$ was determined in Ref. [10] by this route, which is somewhat higher than the usual literature values. The figure shows that the $T=1.3365$ isotherm is flat within statistics over the density range 0.28 and 0.34 and therefore very close to the critical value for the system parameters, $N=4000$ and $r_{c}=2.5$. Tab. 1 lists the pressure along the critical isotherm for $T=1.3365$, which is $0.1406 \pm 0.0002$ for densities between 0.28 and 0.34 . The chemical potential is $-4.136 \pm 0.001$ in the same density range along the critical isotherm, as revealed in the table.

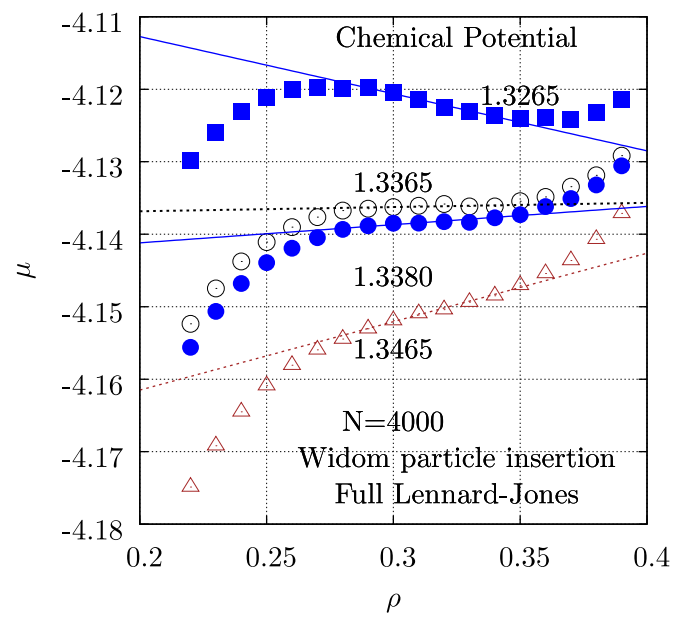

Fig. 5. As for Fig. 4 except that the total chemical potential, $\mu$, obtained by Widom's method against $\rho$ for four isotherms, $T=1.3265,1.3365,1.3380$ and 1.3465 (top to bottom curves, respectively) using the full Lennard-Jones potential is shown. The symbols are the Molecular Dynamics (MD) simulation data points and the lines are linear regression fits, with slopes, $-0.079 \pm 0.005$, $0.006 \pm 0.005,0.025 \pm 0.005$, and $0.094 \pm 0.004$, respectively, for the data curves from top to bottom. The statistically zero slope again occurs at a temperature of $c a .1 .3365$.
Fig. 5 shows the corresponding plot for the chemical potential, $\mu$, determined by Widom's particle insertion method, [17]. The two sets of curves in Figs. 4 and 5 look remarkably similar except that for the chemical potential the temperature decreases from the bottom to top curve. The chemical potential is a linear function of density for temperatures just above and below $T_{c}$ in the critical data 'gap'. The critical temperature is defined by the temperature at which this slope is equal to zero in this density range. The calculated critical temperature for $N=4000$ and $r_{c}=2.5$ is statistically the same as for the $P$ data. Therefore both figures and Tab. 1 are consistent with a 'gap' in physical state of the two coexisting phases at $T_{c}$, rather than a critical point description. The advantage of, in addition, plotting the chemical potential, $\mu$, against density is that the pressure is derived from $\mu$ so this function (which cannot be measured directly by experiment) is a more fundamental one. As $(\partial \mu / \partial P)_{T}=1 / \rho$ then if $P(\rho)$ is a constant in a region of density along the critical isotherm, so must be $\mu(\rho)$.

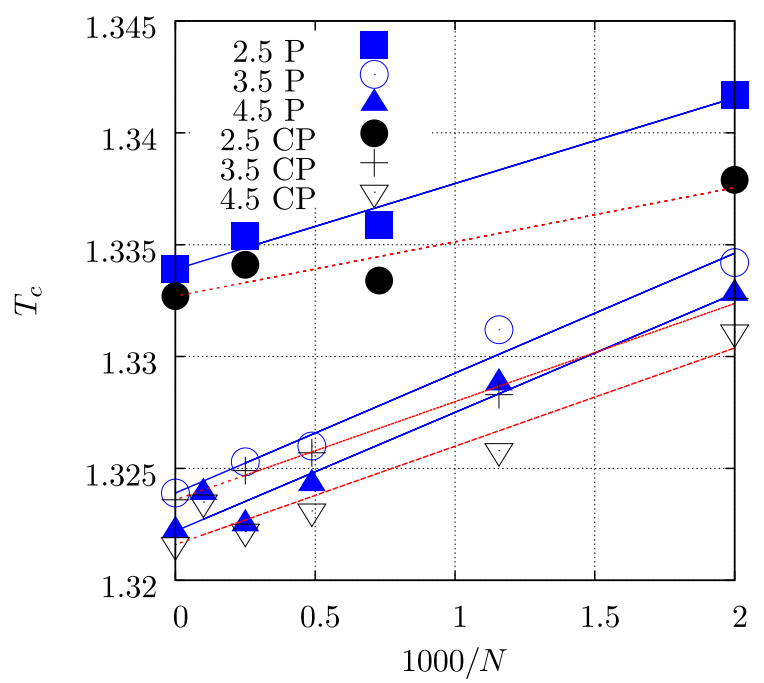

Fig. 6. The critical temperature as a function of the number of particles in the simulation cell, $N$. The sets of data are for the three values of $r_{c}$ and whether the pressure, $P$ or the chemical potential, $C P$, were used to calculate $T_{c}$.

\begin{tabular}{ccccccccc}
\hline$\rho$ & $P$ & $\mu$ & $\rho$ & $P$ & $\mu$ & $\rho$ & $P$ & $\mu$ \\
\hline 0.22 & 0.1366 & -4.152 & 0.28 & 0.1405 & -4.137 & 0.34 & 0.1408 & -4.136 \\
0.23 & 0.1377 & -4.147 & 0.29 & 0.1406 & -4.136 & 0.35 & 0.1408 & -4.135 \\
0.24 & 0.1387 & -4.144 & 0.30 & 0.1406 & -4.136 & 0.36 & 0.1408 & -4.135 \\
0.25 & 0.1393 & -4.141 & 0.31 & 0.1407 & -4.136 & 0.37 & 0.1411 & -4.133 \\
0.26 & 0.1399 & -4.139 & 0.32 & 0.1406 & -4.136 & & & \\
0.27 & 0.1403 & -4.138 & 0.33 & 0.1406 & -4.136 & & & \\
\hline
\end{tabular}

Tab. 1. Density dependence of the pressure, $P$ and chemical potential, $\mu$, obtained by Widom's particle insertion method for $N=4000$ Lennard-Jones particles at the critical temperature, $T=1.3365$ for the $r_{c}=2.5$ system. Each density state point was conducted for 4 million time steps. The standard errors in the pressure and chemical potential are \pm 1 in the last digit. 


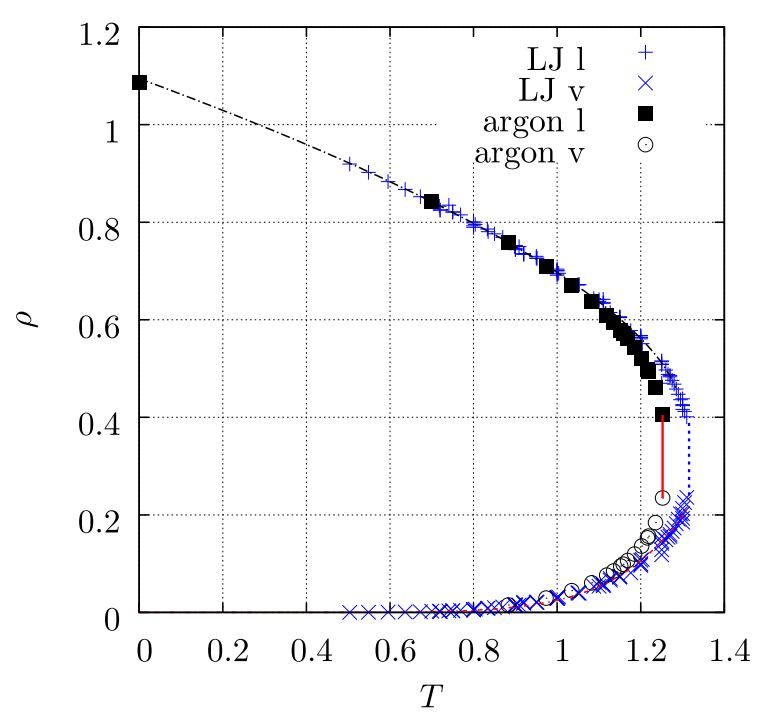

Fig. 7. The liquid-vapour coexistence envelope obtained from molecular simulation (see the references listed in Fig.1) compared with that of argon scaled by the LJ parameters, $\sigma=0.3405 \mathrm{~nm}$ and $\left.\epsilon / k_{B}=119.8\right)$, [34, 35]. Both sets of data are in LJ reduced units. The simulation data were fitted to, $\rho_{l}=a_{l}+b_{l}\left(T_{c l}-T\right)^{c_{l}}$, where $a_{l}=0.431241, b_{l}=0.566753, c_{l}=0.614399$ and $T_{c l}=$ 1.291639 for the liquid side of the binodal, and $\rho_{v}(x)=b_{v} T^{c_{v}}$, where $b_{v}=0.0251177$ and $c_{v}=7.880728$ for the vapour side of the binodal. These are the continuous lines going through the data on the figure. For the liquid part of the binodal the intercept value was calculated based on random close packing of spheres, $\rho_{f}=0.948$ and $\rho_{R C P}=1.218$, and the triple point density of argon of 1417 $\mathrm{kg} \mathrm{m}^{-3}$ [34], which is shown as the solid symbol on the ordinate axis at $c a$. 1.1. The vertical lines on the right side of the figure mark out the regions of absence of data for the model and real systems. The symbols, ' $l$ ' and ' $v$ ' on the figure refer to liquid and vapour, respectively.

The results in Figs. 4 and 5 are for a specific value of $N$ and $r_{c}$. The critical temperature was determined for each value of $r_{c}$ and $N$ by carrying out many simulations over the relevant density range at various temperatures. The temperature at which the slope of $P$ against $\rho$ is zero was obtained by linear interpolation of the slopes found for the different temperatures. The values of these $r_{c^{-}}$and $N$-dependent $T_{c}$ are plotted as a function of $1 / N$ in Fig. 6 . For each value of $r_{c}$ these sets of data were fitted by linear regression and the value of $T_{c}$ in the $N \rightarrow \infty$ or thermodynamic limit was thereby determined. These are indicated by the intercepts or $N^{-1}=0$ value of $T_{c}$ on this figure, whose values are given in Tab. 2. Lacking any definitive extrapolation procedure, the value of $T_{c}$ in the $r_{c} \rightarrow \infty$ or thermodynamic limit was obtained by fitting these intercept data to the formula, $T_{c}\left(r_{c}\right)=T_{c}(\infty)+A / r_{c}^{m}$ for the cases $m=1,2$ and 3 . This is a generalisation of the formula used by Dunikov et al. [21]. The value of $T_{c}(\infty)$ for each value of $m$ is given in the bottom three rows of Tab. 2, i.e. $P_{1}, P_{2}$ and $P_{3}$, respectively. The above procedure was also carried out for the chemical potential data. The critical temperature decreases with increasing $r_{c}$, and taking the $m=2$ or middle exponent value gives a value of the $T_{c}$ of $1.316 \pm 0.002$ in the $N \rightarrow \infty$ and $r_{c} \rightarrow \infty$ limits using the pressure data, and $T_{c}=1.316 \pm 0.001$ using the chemical potential data. Within statistics this is almost the same as typical values found in the literature for example, $T_{c}=1.3120(7),[22,23]$. It may be concluded therefore that the critical temperature is sensitive to the direct LJ force truncation distance, even when the Morris-Song additional taper is patched on to the $\mathrm{LJ}$ potential at $r=r_{c}$. The range of $T_{c}$ values is however much smaller and closer to the thermodynamic limit value of $T_{c}$ for each $r_{c}$ used than that found by straightforward truncation of the potential at $r_{c}$ in Ref. [21].

To summarize, these data and trends explain the relatively high value of $T_{c}$ found in Ref. [10]. The simulation data from each value of $r_{c}$ is a bona fide physical system in its own right nevertheless, to which all the phase behaviour associated with liquid-vapour criticality should apply.

Also shown in Tab. 2 is the value of the $\mathrm{LJ}$ critical point for various $r_{c}$ values taken from the work of Dunikov et $a l$. [21] who truncated the pair potential at $r_{c}$. They found that $T_{c}$ increased with $r_{c}$ whereas the opposite trend is found using the present method of potential long range treatment. Their value of $T_{c}$ for infinite $r_{c}$ is 1.34 (see Eq. (21) in Ref. [21]), which is rather large compared to other literature values. These two works, combined, show that $T_{c}$ can either decrease or increase with $r_{c}$ depending on how the

\begin{tabular}{cccccc}
\hline$r_{c}$ & Property & $T_{c}$ & Property & $T_{c}$ & $T_{c}^{\prime}$ \\
\hline 2.5 & $P$ & $1.334(1)$ & $\mu$ & $1.333(1)$ & $1.085(5)$ \\
3.5 & $P$ & $1.324(1)$ & $\mu$ & $1.324(1)$ & $1.213(2)$ \\
4.5 & $P$ & $1.322(1)$ & $\mu$ & $1.322(1)$ & - \\
$\infty$ & $P_{1}$ & $1.306(5)$ & $\mu_{1}$ & $1.307(4)$ & 1.34 \\
$\infty$ & $P_{2}$ & $1.316(2)$ & $\mu_{2}$ & $1.316(1)$ & 1.34 \\
$\infty$ & $P_{3}$ & $1.319(1)$ & $\mu_{3}$ & $1.319(1)$ & 1.34 \\
\hline
\end{tabular}

Tab. 2. The critical temperature, $T_{c}$ as a function of the $\mathrm{LJ}$ interaction termination distance, $r_{c}$, obtained from the data of this work. This procedure patches on a tapered decay of the potential to zero for $r>r_{c}$ from $r_{c}$ to $r_{c}+0.2$. Also shown is the prediction from Ref. [21], which is denoted by, $T_{c}^{\prime}$, and is based on a truncation of the interaction at $r_{c}$. The number in brackets is the statistical uncertainty in the last digit. The extrapolated infinite $r_{c}$ truncation value based on data in the first three rows using, $T^{c}\left(r_{c}\right)=T_{c}(\infty)+A / r_{c}^{m}$ are given for $m=1,2$ and 3 in the last three rows, i.e. $P_{1}, P_{2}$ and $P_{3}$, respectively. The corresponding quantities for the chemical potential are, $\mu_{1}, \mu_{2}$ and $\mu_{3}$. 
'missing' interactions beyond $r_{c}$ are dealt with.

Fig. 7 shows the liquid-vapour coexistence line obtained from molecular simulation (see the references listed in Fig. 1) compared with that of argon using the LJ parameters, $\sigma=$ $0.3405 \mathrm{~nm}$ and $\left.\epsilon / k_{B}=119.8 \mathrm{~K}\right),[34,35]$. Both sets of data are plotted in LJ reduced units. Argon is the small molecule which is thought to be best represented by the LJ potential. Least square fits to the liquid and vapour sides of the simulation data separately are shown on the figure as continuous lines with functional forms given in the figure caption. The vertical lines on the right side of the figure mark out where there is a gap in data for model and real systems, which are seen to be of similar length. At low density both the simulation and argon data agree well, but on approaching the critical region the experimental data terminate at a lower value for the critical temperature (using the above conversion factors), which may be a consequence of the three body and higher order terms present in the real system. A decrease in $T_{c}$ by ca. $95 \%$ on inclusion of three body forces compared to that of the two-body LJ potential has been observed in previous simulation studies, [36-38]. The value of $T_{c}$ obtained with the three-body terms in those simulations agrees well with the $T_{c}$ of experimental argon. This trend can also be inferred from recent simulations of Goujon et al. [39-41], which showed that the surface tension systematically decreases at all temperatures on adding the three body potential to the two-body potential term.

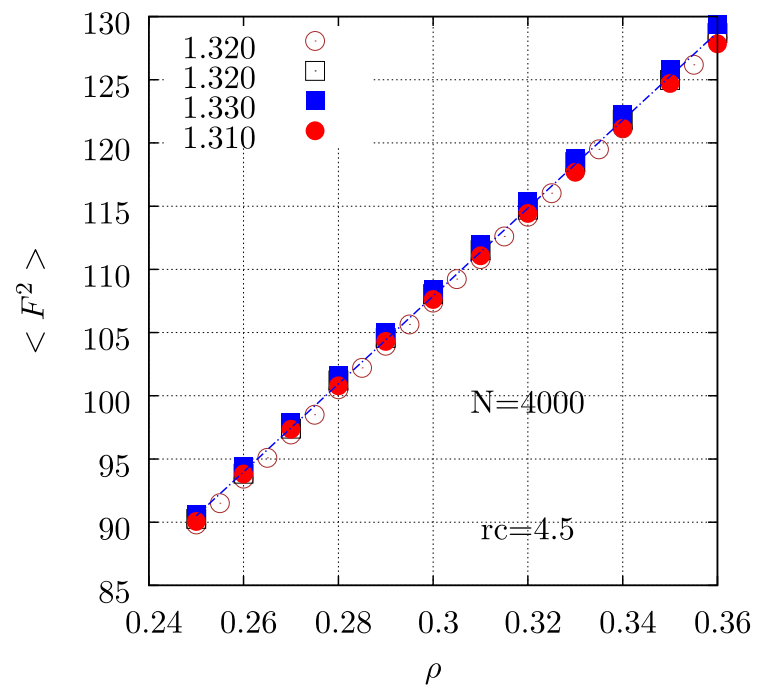

Fig. 8. Mean square force, $\left\langle F^{2}>\right.$ as a function of number density, $\rho$, for $N=4000$ and $r_{c}=4.5$ using isotherms close to $T_{c}$. A least squares fit to the data of the $T=1.320$ isotherm to the form, $<F^{2}>=a+b \rho$ gives, $a=3.57$ and $b=348$. The direct pair interaction termination distance, $r_{c}$ is denoted by ' $r c$ ' on this and subsequent figures

The liquid part of coexistence in the low temperature limit shows another noteworthy feature. Does the finite value of $\rho_{l}$ by extrapolating the liquid side of the binodal to $T \rightarrow 0$ have any significance for our understanding of the liquid state, even though it is in the metastable liquid part of the phase diagram? For the liquid part of the binodal the intercept value was calculated based on random close packing (RCP) of spheres, which is shown as a symbol on the ordinate axis. The maximum equilibrium fluid density of hard spheres is, $\rho_{f}=0.948$ and the RCP density, $\rho_{R C P}=1.218$. If the triple point density of argon, $1417 \mathrm{~kg} \mathrm{~m}^{-3}$ [34] is taken to correspond to $\rho_{f}$, the following conclusion can be drawn. The extrapolated value of both the simulation and experimental argon data in the zero temperature limit (i.e. $\rho=1.0945$ in LJ units) coincides remarkably well with the corresponding (estimated) RCP value of argon for the LJ system. This result is consistent with the conclusions of Finney and Woodcock in Ref. [43] who argued that the random close packing point of hard spheres should be considered to be the statistical mechanical foundation of the liquid state.

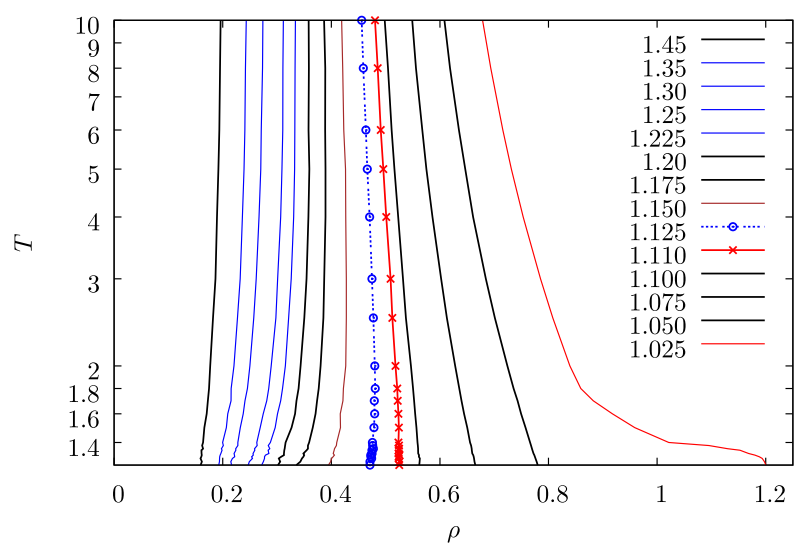

Fig. 9. The lines of equal percolation threshold distance, $r_{p}$, given on the temperature-density phase diagram. Note the lin-log plot

The variations of other properties in the critical density range and along isotherms close to the critical temperature are now considered. Fig. 8 shows the mean square force, $<F^{2}>$, on a molecule as a function of density along several isotherms close to the critical temperature for systems with $N=4000$ and $r_{c}=2.5$. A near linear dependence is apparent in the figure. The mean square force increases by ca. $50 \%$ on going from $\rho=0.24$ to 0.36 , which is the largest change of all the properties in this density range near $T_{c}$ (see below for the variation of other properties in this $\rho$ range). A least squares fit to the data of the $T=1.320$ isotherm to the form, $<F^{2}>=a+b \rho$ gives, $a=3.57$ and $b=348$. It is not obvious what the origin of this linear behaviour is. It might reflect a progressive shift from a vapour/gas-like to a liquid-like phase in a mixed phase region with increasing density, as argued in Ref. [10]. A transition between two different regimes of the density-dependence of the maximum single particle force of soft-sphere systems has been observed (see Fig. 3 in Ref. [42]), which may be related to this trend. The single particle or pair interaction force distribution could 
be a useful (largely unexploited) probe of the presence of physically meaningful percolating networks of relevance to liquid-vapour criticality. Indeed the importance of percolating force networks is already well-established in the granular materials community.

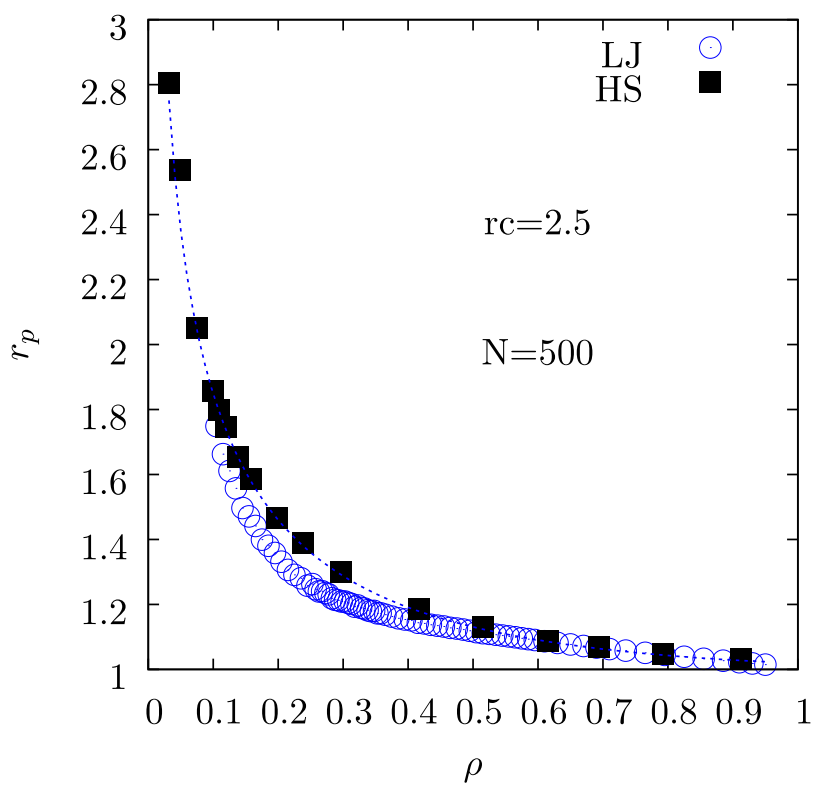

Fig. 10. The percolation distance for $T=1.3365$ and $r_{c}=2.5$. The square symbols are the MD data for hard spheres (HS) of Ref. [44]. For the HS calculations the effective hard-sphere diameter was obtained from Eq. (9) in Ref. [49], and Eqs. (6), (9) and (10) of Ref. [44] were used to produce the continuous line on the figure

The microstructure of simple fluids is usually characterised in terms of the radial distribution function, $g(r)$. This function is however insensitive to clustering behaviour which may be important in providing a better understanding of the critical region. Percolation is an interparticle connectivity transition of the state of particle clusters which can have physical consequences (e.g. conduction and rigidity transitions) for real systems such as colloidal liquids and composites. The determination of the percolation statistics for various pair potential forms has already been performed by molecular simulation. In one such study, [44], the percolation distance, $r_{p}$, was calculated for hard-sphere fluids of sphere diameter, $\sigma_{H S}$, in the range between the so-called soft-core $\left(r_{p}>>\sigma_{H S}\right)$ to hard-core $\left(r_{p} \rightarrow \sigma_{H S}\right)$ limits, both of which are of relevance to this work. The same procedure for determining $r_{p}$ was used in the present study. The number density at which percolation occurs for a given value of $r_{p}$ is here referred to as, $\rho_{p}$.

Fig. 9 shows the temperature dependence of $\rho_{p}$ for a set of $r_{p}$ which span the soft-core to hard-core limits. These iso- $r_{p}$ percolation lines on the figure at low density curve to the left on decreasing temperature. For smaller values of $r_{p}$ on the right side of the critical region, the percolation lines curve to the right as temperature decreases, and some even cross into the solid region of the phase diagram as is evident on the figure by the sharp horizontal jump of the $r_{p}=1.025$ line. In the range, $r_{p} \simeq 1.11-1.125$ the percolation line is essentially independent of temperature, which therefore acts as a boundary between two qualitatively different types of behaviour. One may speculate that this is associated with the shift outwards in the first peak in $g(r)$ (in particular the $r$ value where the first non-zero value appear) on going from fluid to co-existing solid.

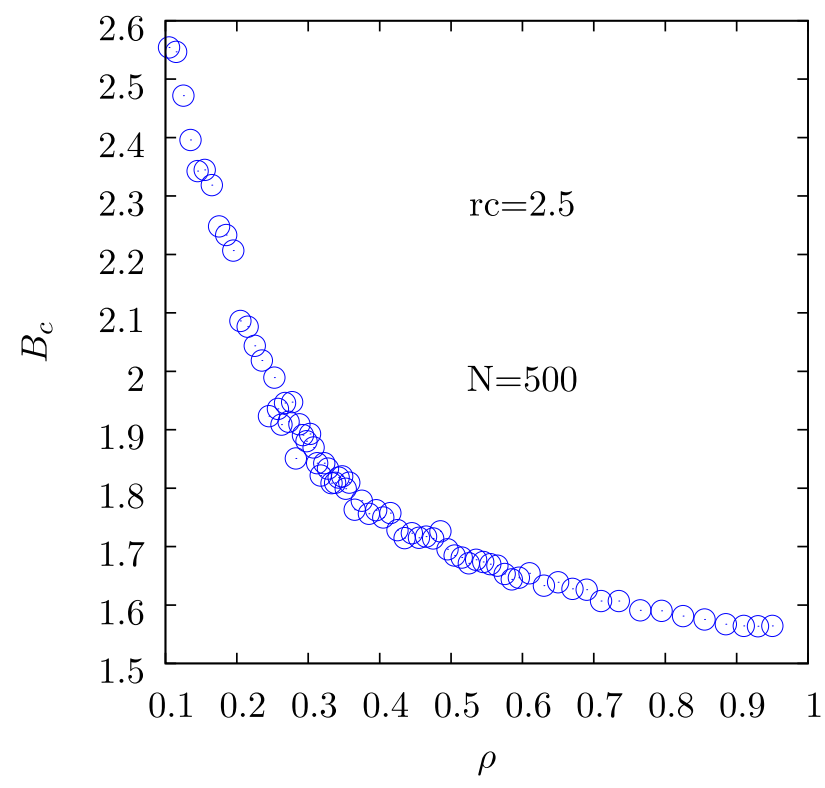

Fig. 11. The average coordination number, $B_{c}$ as a function of density, $\rho$ for the same systems as in Fig. 10

The Monte Carlo simulations by Bug et al. [45] on square wells revealed that at low density (or equivalently large $r_{p}$ ) the effect of attraction with decreasing temperature is to cause the clusters to become more compact, presumably for energetic reasons to maximise the overlap of the soft shells. This initially produces an increase in $\rho_{p}$, but at state points close to the liquid-vapour binodal the value of $\rho_{p}$ starts to decrease as $T$ decreases. The square wells also show on the right side of the coexistence region a decrease in $\rho_{p}$ with decrease in temperature, [46-48]. Therefore the behaviour of the LJ system is qualitatively different to the square wells in certain aspects, in that the $\mathrm{LJ} \rho_{p}$ always decreases with decrease in temperature on the left-side of the critical region and it always increases on the right side. This suggests that for the LJ fluid state, on the left side of coexistence the effect of the attraction is to enhance the connectivity of the particles by reorganizing to a more elongated clusters which more readily percolate at a given particle number density. On the right side of the critical region there is a decrease in the average number of neighbours in a percolating cluster for excluded volume reasons, which also may make percolation less likely, with a percolating cluster which is more 'tenuous' than that found at low densities. One cannot rule out that this shift to the right 


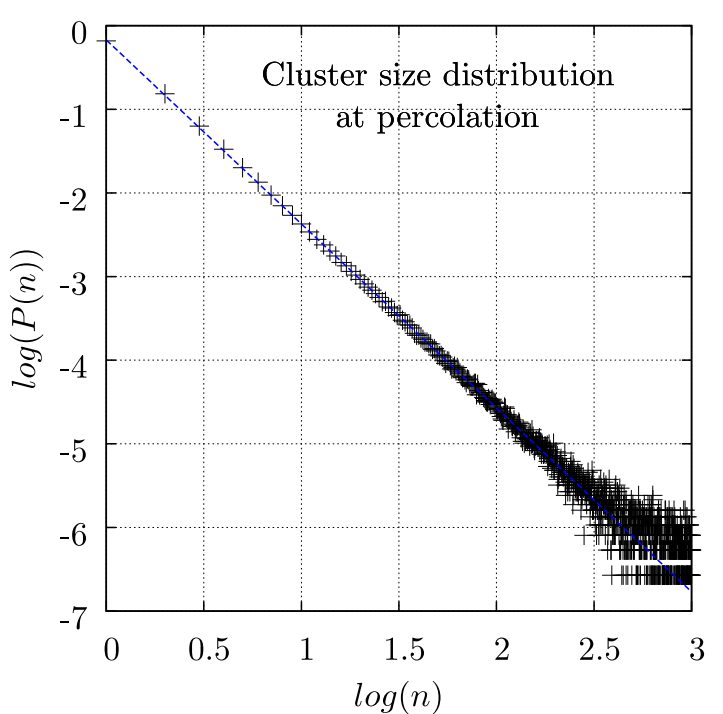

Fig. 12. Cluster number distribution function at percolation, $P(n)$, where $n$ is the number of atoms in the cluster for the LJ state point $T=1.3365, \rho=0.40, N=4000, r_{c}=2.5$ and $r_{p}=1.15$. The $\log$ is $\log _{10}$

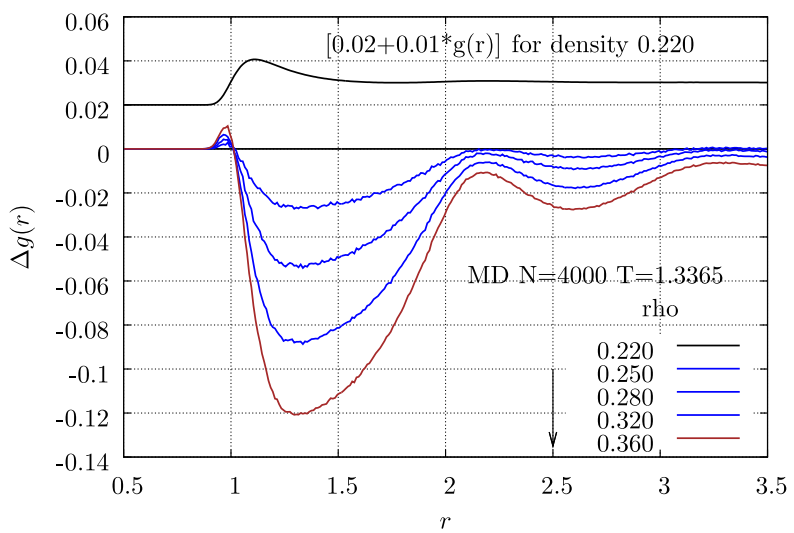

Fig. 14. The top curve is the radial distribution function, $g(r)$ for $T=1.3365, N=4000, r_{c}=2.5$ and $\rho=0.220$. The lower four curves are $\Delta g(\rho, r)=g(\rho, r)-g(\rho=0.220, r)$ to highlight how $g(r)$ changes with density along the flat part of the critical isotherm

of $\rho\left(r_{p}\right)$ at high densities in the liquid region for the states bordering on the fluid-solid boundary contains an aspect of 'pre-transitional anticipation' of the impending solid phase in the inherent structures.

Fig. 10 shows the percolation distance along the critical isotherm, $T=1.3365$ and $r_{c}=2.5$. The square symbols are the MD data for hard spheres (HS) taken from Ref. [44]. For the HS calculations the effective hard-sphere diameter was obtained from Eq. (9) in Ref. [49], and Eqs. (6), (9) and (10) of Ref. [44] were used to produce the continuous line

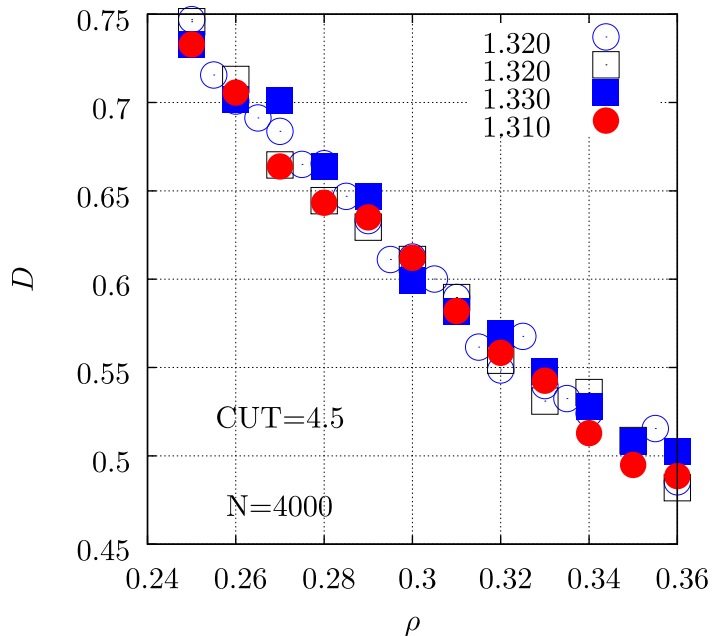

Fig. 13. The self-diffusion coefficient, $D$, as a function of $\rho$ for several temperatures close to $T_{c}$, which are given on the figure

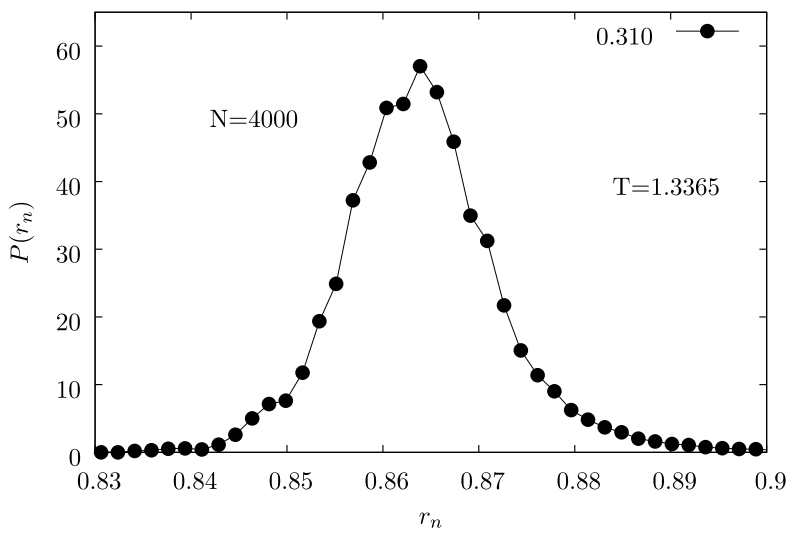

Fig. 15. Histogram of the nearest neighbour distance, $r_{n}$ for $\rho=0.310$ in the flat part of the critical isotherm. The other parameters are: $T=1.3365, N=4000$ and $r_{c}=2.5$

on Fig. 10. It is evident that the LJ data agree with the MD curves at high and low density, but at intermediate densities the LJ percolation distance falls below that of the HS case as previously commented on in relation to Fig. 9.

Fig. 11 shows the average coordination number per particle, $B_{c}$, (or average number of neighbours within $r_{p}$ about an arbitrary particle) as a function of the density, $\rho$, for the same state points as in Fig. 10. This quantity decreases from 2.8 to 1.6 from $\rho \rightarrow 0$ to the dense fluid limit, respectively. The same quantitative behaviour is found for hard-spheres [44]. 


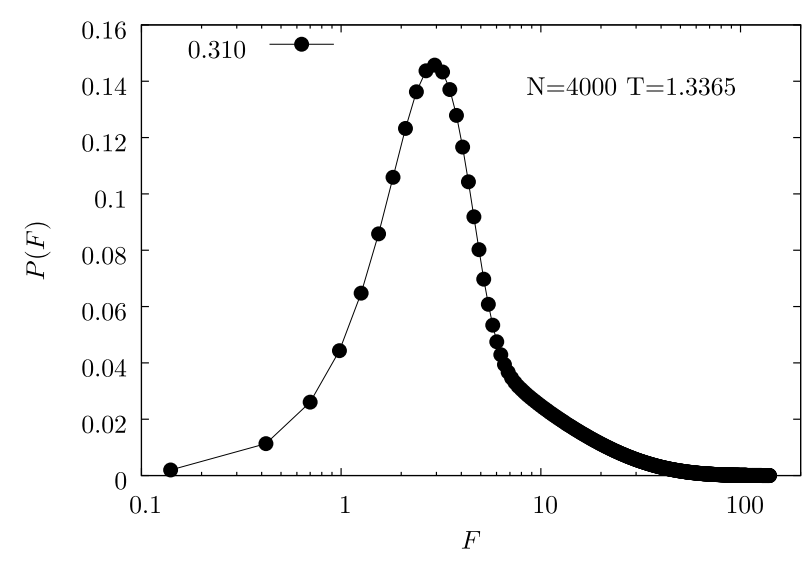

Fig. 16. As for Fig. 15 except the probability distribution of the absolute or net force on a particle, $F$ is shown. Note the log-lin scale

Fig. 12 presents a representative cluster distribution function at percolation, $P(n)$, where $n$ is the number of atoms in the cluster. At percolation $P(n) \sim n^{-\tau},[50,51]$ and the exponent determined by least squares fitting is $\tau=$ $2.200 \pm 0.006$ which is in excellent agreement with the 3D lattice value of $2.2[50,51]$.

Fig. 13 shows the density dependent self-diffusion coefficient, $D$, obtained by linear regression of the mean square displacements of molecules for near critical temperature states at densities in the critical region. As with the mean square force, it is nearly linear but this quantity decreases with increasing density. Fig. 14 shows the radial distribution function, $g(r)$, for $T=1.3365$ and $\rho=0.220$ at the top of the figure. The difference between this and $g(r)$ at higher densities in the critical region (the lower set of curves) is also shown in the figure. It can be seen that with increasing density there is an increase in the average number of neighbours at very short range but a decrease at longer range.

Figures 15-17 present probability distribution functions of the nearest neighbour separation, $r_{n}$, the total absolute force on a molecule, $F$ and the potential energy of a molecule, $u$, respectively. They are quite similar in shape, except that the force distribution function shows a slowly decaying tail at the high force end. A more complete description of the critical region should include the average effects of the fluctuations in certain quantities.

\section{CONCLUSIONS}

This work continues a study started in Ref. [10] in providing an analysis of various properties of the Lennard-Jones fluid in the liquid-vapour critical region. It has been shown that the origin of the relatively high critical point temperature determined previously compared to previous studies is attributable to the value of the maximum range used to

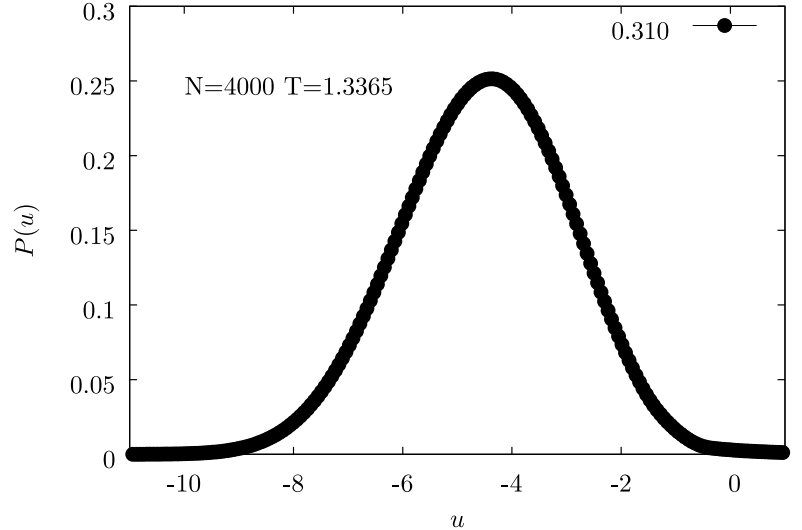

Fig. 17. As for Fig. 15 except that the probability distribution of the potential energy of a particle, $u$, of the critical isotherm is shown

calculate the pair interactions directly with the original $\mathrm{LJ}$ potential. The range of the 'cut-off' distance on $T_{c}$ is significant, and even for $r_{c}=4.5$ reasonable convergence to the infinite $r_{c}$ limit is still not achieved. Of relevance to this conclusion are Molecular Dynamics results, [52] which show that only for truncation distances in excess of $5.5 \sigma$ do the thermodynamic properties converge to the value obtained by an Ewald method route (which formally includes the interactions from all molecules in the periodic system, but has system size limitations in practice). It has also been found that the solid-liquid coexistence properties for truncated, and truncated and shifted potentials vary systematically and only with a truncation distance of $6.5 \sigma$ is consistency obtained between the different treatments, [53].

A more detailed analysis of the percolation properties has been made. Whether the critical point or 'flat top' descriptions of criticality is correct is not going to change the fact that the density dependence of the pressure along the critical isotherm is extremely flat, [15]. In fact, for most practical applications it can be taken to be flat to a very good approximation. The precise shape of this region will however have major implications for what the phase diagram in the supercritical region is, and whether or not it is divided into a gas-like, mesophase, and liquid-like domains. If the flat-top description is correct it would also change our understanding of what is meant by a 'liquid', [43]. To date, neither simulation nor experiment has conclusively demonstrated which picture is correct. One feature of small molecule phase diagrams found in many textbooks is the appearance of a continuous path between liquid and vapour phases via the supercritical region without any apparent phase boundaries separating these three phases. This alone would suggest a reconsideration of the widely accepted theory of a criticality in fluid phase equilibria is timely. The critical point also does not satisfy Gibbs' phase rule as it is not the intersection of two two-phase coexistence lines, as is the case for the triple point. 


\section{References}

[1] T. Andrews, On the continuity of the gaseous and liquid states of matter, Phil. Trans. R. Soc. Lond. 159, 575-590 (1869).

[2] A.A. Mills, The critical transition between the liquid and gaseous conditions of matter, Endeavour 19, 69-75 (1995).

[3] J.D. van der Waals: On the continuity of the gas and liquid state, $\mathrm{Ph} . \mathrm{D}$. Thesis (University of Leiden, The Netherlands, 1873).

[4] E.A. Guggenheim, The principle of corresponding states, J. Chem. Phys. 13, 253-261 (1945).

[5] B. Widom and O.K. Rice, Critical isotherm and the equation of state of liquid vapour systems, J. Chem. Phys. 23, 1250-1255 (1955).

[6] N.S. Barlow, A.J. Schultz, D.A. Kofke, and S.J. Weinstein, Critical isotherms asymptotically consistent approximants, AIChE Journal, 60, 3336-3349 (2014).

[7] S.F. Harrison and J.E. Mayer, Statistical mechanics of condensing systems. IV, J. Chem. Phys. 6, 101-104 (1938).

[8] L.V. Woodcock, Thermodynamic description of liquid-state limits, J. Phys. Chem. B, 116, 3735-3744 (2012) .

[9] L.V. Woodcock, Fluid phases of argon: A debate on the absence of van der Waals' "critical point", Natural Science, 5, 194-206 (2013).

[10] D.M. Heyes and L.V. Woodcock, Critical and supercritical properties of Lennard-Jones fluids, Fluid Phase Equil., 356, 301-308 (2013).

[11] J.M.H. Levelt Sengers, Liquidons and gasons. Controversies about the continuity of states, Physica A, 98, 363-402 (1979).

[12] L.P. Kadanoff, More is the same; phase transitions and mean field theories, J. Stat. Phys., 137 777-797 (2009).

[13] R.Gilgen, R. Kleinrahm and W. Wagner, Measurement and correlation of the (pressure, density, temperature) relation of $\operatorname{argon} \mathrm{I}$. The homogeneous gas and liquid regions in the temperature range from $90 \mathrm{~K}$ to $340 \mathrm{~K}$ at pressures up to $12 \mathrm{MPa}$, J. Chem. Therm. 26, 383-398 (1994).

[14] R.Gilgen, R. Kleinrahm and W. Wagner, Measurement and correlation of the (pressure, density, temperature) relation of argon II. saturated-liquid and saturated-vapour densities and vapour pressures along the entire coexistence curve. J. Chem. Therm. 26, 399-413 (1994).

[15] K. Rah and B.C. Eu, Phenomenological models for the generic van der Waals equation of state and critical parameters, J. Phys. Chem. B 107, 4382-4391 (2003).

[16] D.M. Heyes, Liquids at positive and negative pressure, Phys. Stat. Solidi B 245 (2008) 530-538.

[17] B. Widom, J. Phys. Chem. 39, 2808-2812 (1963).

[18] J.R. Morris and X. Song, J. Chem. Phys., The melting lines of model systems calculated from coexistence simulations, 116, 9352-9358 (2002).

[19] D. Heyes, The Liguid State - Applications of Molecular Simulations, J. Wiley \& Sons, Chichester 1998.

[20] H. Watanabe, N. Ito and C.-K. Hu, Phase diagram and universality of the Lennard-Jones gas-liquid system, J. Chem. Phys., 136, 204102 (2012).

[21] D.O. Dunikov, S.P. Malyshenko and V.V. Zhakhovskii, Corresponding states law and molecular dynamics simulations of the Lennard-Jones fluid, J. Chem. Phys. 115, 6623-6631 (2001) .

[22] J.J. Potoff and A.Z. Panagiotopolous, Surface tension of the three-dimensional Lennard-Jones fluid from histogramreweighting Monte Carlo simulations, J. Chem. Phys. 112, 6411-6415 (2000).

[23] J.Pérez-Pellitero, P. Ungerer, G. Orkoulas and A.D. Mackie, Critical point estimation of the Lennard-Jones pure fluid and binary mixtures, J. Chem. Phys. 125, 054515 (2006).
[24] J. Mick, E. Hailat, V. Russo, K. Rushaidat, L. Schwiebert and J. Potoff, GPU-accelerated Gibbs ensemble Monte Carlo simulations of Lennard-Jonesium, Comp. Phys. Comm. 184, 2662 (2013).

[25] J. Kolafa and I, Nezbeda, The Lennard-Jones fluid: An accurate analytic and theoretically-based equation of state, Fluid Phase Equilibria 100, 1-34 (1994).

[26] M.A. Barroso and A.L. Ferreira, Solid fluid coexistence of the Lennard-Jones system from absolute free energy calculations, J. Chem. Phys. 116, 7145-7150 (2002) .

[27] V.G. Baidakov, S.P. Protsenko and Z.R. Kozlova, Metastable Lennard-Jones fluids. I. Shear viscosity, J. Chem. Phys. 137, 164507 (2012).

[28] A. Lofti, J. Vrabec and J. Fischer, Vapour liquid equilibria of the Lennard-Jones fluid from the NpT plus test particle method, Mol. Phys. 76, 1319-1333 (1992).

[29] D.A. Kofke, Direct evaluation of phase coexistence by molecular simulation via integration along the saturation line, J. Chem. Phys. 98 4149-4162 (1993) .

[30] A. Trokhymchuk and J. Alejandre, Computer simulations of liquid/vapour interface in Lennard-Jones fluids: Some questions and answers, J. Chem. Phys. 111, 8510-8523 (1999).

[31] H. Okumura and F. Yonezawa, Liquid vapour coexistence curves of several interatomic model potentials, J. Chem. Phys. 113, 9162-9168 (2000).

[32] H. Okumura and F. Yonezawa, Reliable determination of the liquid-vapour critical point by the NVT plus test particle method, J. Phys. Soc. Japan 70, 1990-1994 (2001).

[33] S. Reif-Acherman, The history of the rectilinear diameter law, Quimica Nova. 33 2003-2010 (2010).

[34] Ch. Tegeler, R. Span and W. Wagner, A new equation of state for argon covering the fluid region for temperatures from the melting line to $700 \mathrm{~K}$ at pressures up to $1000 \mathrm{MPa}$, J. Phys. Chem. Ref. Data, 28, 779-850 (1999).

[35] L. A. Rowley, D. Nicholson and N. G. Parsonage, Monte Carlo grand canonical ensemble calculation in a gas-liquid transition region for 12-6 Argon, J. Comput. Phys. 17 401-414 (1975).

[36] R. J. Sadus, Exact calculation of the effect of three-body Axilrod-Teller interactions on vapour-liquid phase coexistence, $\mathrm{Fl}$. Phas. Equil. 144 351-359 (1998).

[37] R.Bukowski and K. Szalewicz, Complete ab initio threebody nonadditive potential in Monte Carlo simulations of vapour-liquid equilibria and pure phases of argon , J. Chem. Phys. 114, 9518-9531 (2001).

[38] J. A. Anta, E. Lomba, and M. Lombardero, Influence of threebody forces on the gas-liquid coexistence of simple fluids: the phase equilibrium of argon, Phys. Rev. E. 55, 2707-2712 (1997).

[39] F. Goujon, P. Maffreyt and D.J. Tildesley, The gas-liquid surface tension of argon: A reconciliation between experiment and simulation, J. Chem. Phys. 140, 244710 (2014).

[40] S. Werth, M. Jorsch, J. Vrabec and H. Hasse, Comment on "The gas-liquid surface tension of argon: A reconciliation between experiment and simulation", J. Chem. Phys. 142, 107101 (2015).

[41] F. Goujon, P. Maffreyt and D.J. Tildesley, Response to "Comment on 'The gas-liquid surface tension of argon: A reconciliation between experiment and simulation", J. Chem. Phys. 142, $107102(2015)$.

[42] G. Rickayzen, A.C. Brańka, S. Pieprzyk and D.M. Heyes, Single particle force distributions in simple fluids, J. Chem. Phys. 137, 094505 (2012).

[43] J.L. Finney and L.V. Woodcock, Renaissance of Bernal's random close packing and hypercritical line in the theory of liquids, J. Phys. Cond. Matt. 26463120 (2014). 
[44] D.M. Heyes, M. Cass and A.C. Bránka, Percolation threshold of hard-sphere fluids in between the soft-core and hard-core limits, Mol. Phys. 104, 3137-3146 (2006) .

[45] A.L.R. Bug, S.A. Safran, G.S. Grest and I. Webman, Do interactions raise or lower a percolation threshold?, Phys. Rev. Lett. 55, 1896-1899 (1985).

[46] D.M. Heyes, Cluster analysis and continuum percolation of 3D square-well phases: MC and PY olutions, Mol. Phys. 69, 559-569 (1990).

[47] D.M. Heyes and J.R. Melrose, Percolation cluster statistics of Lennard-Jones fluids, Mol. Phys. 66, 1057-1074 (1989) .

[48] D.M. Heyes, Monte Carlo Simulations of continuum percolation of 3D well fluids, J. Phys. Cond. Matt. 2, 2241-2249 (1990).

[49] D.M. Heyes and H. Okumura, Equation of state and structural properties of the Weeks-Chandler-Andersen fluid, J. Chem. Phys. 124, 164507 (2006).

[50] D. Stauffer, Scaling theory of percolation clusters, Phys. Rep. 54, 1-74 (1979).

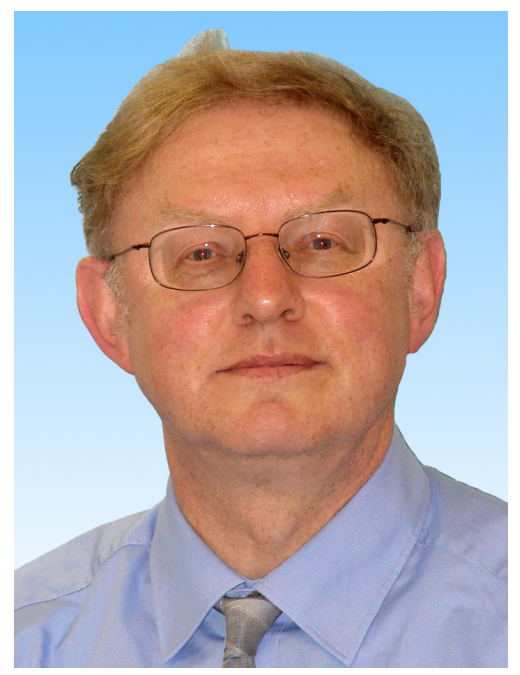

David Heyes [PhD, University of Manchester, UK, 1977] has held postdoctoral research positions at the Vitreous State Laboratory, Department of Physics, Catholic University of America, Washington DC, USA, Department of Physical Chemistry at the University of Amsterdam, The Netherlands, and at the Department of Chemistry, Royal Holloway, University of London, UK. He was a Royal Society (London) 1983 University Research Fellow between 1983 and 1992 first at Royal Holloway and then at the University of Surrey UK. After holding a faculty position at the University of Surrey he moved to the Department of Physics, Royal Holloway as Professor of Physics. He is a Principal Research Fellow in the Department of Mechanical Engineering, Imperial College London. His research interests are in the development of computer-based theoretical and modelling techniques applied to liquid systems, principally in the areas of phase equilibria, and structural and transport properties of bulk and confined systems. His current research interests involve carrying out Non-equilibrium Molecular Dynamics (NEMD) simulations of confined systems under shear, developing scaling theories of the static and dynamical properties of molecular liquids, and devising coarse-graining strategies for the electrostatic properties of soft condensed matter systems. 